\title{
Positive Radial Solutions for Singular Quasilinear Elliptic Equations in a Ball
}

\author{
by
}

\author{
Dang Dinh HAI
}

\begin{abstract}
We establish the existence of positive radial solutions for the boundary value problems

$$
\begin{cases}-\Delta_{p} u=\lambda f(u) & \text { in } B \\ u=0 & \text { on } \partial B\end{cases}
$$

where $\Delta_{p} u=\operatorname{div}\left(|\nabla u|^{p-2} \nabla u\right), p \geq 2, B$ is the open unit ball $\mathbb{R}^{N}, \lambda$ is a positive parameter, and $f:(0, \infty) \rightarrow \mathbb{R}$ is $p$-superlinear at $\infty$ and is allowed to be singular at 0 .

2010 Mathematics Subject Classification: 35J75, 35J92.

Keywords: $p$-superlinear, singular, $p$-Laplace, positive solutions.
\end{abstract}

\section{$\S 1$. Introduction}

In this paper, we study the existence of positive radial solutions for the boundary value problem

$$
\begin{cases}-\Delta_{p} u=\lambda f(u) & \text { in } B \\ u=0 & \text { on } \partial B\end{cases}
$$

where $\Delta_{p} u=\operatorname{div}\left(|\nabla u|^{p-2} \nabla u\right), p \geq 2, B$ is the open unit ball $\mathbb{R}^{N}, N>1, \lambda$ is a positive parameter, and $f:(0, \infty) \rightarrow \mathbb{R}$.

Thus we shall consider the ODE problem

$$
\left\{\begin{array}{l}
-\left(r^{N-1} \phi\left(u^{\prime}\right)\right)^{\prime}=\lambda r^{N-1} f(u), \quad 0<r<1, \\
u^{\prime}(0)=0, \quad u(1)=0
\end{array}\right.
$$

where $\phi(z)=|z|^{p-2} z$.

Communicated by H. Okamoto. Received June 27, 2013. Revised November 24, 2013.

D. D. Hai: Department of Mathematics and Statistics, Mississippi State University, Mississippi State, MS 39762, USA;

e-mail: dang@math.msstate.edu

(C) 2014 Research Institute for Mathematical Sciences, Kyoto University. All rights reserved. 
There is a vast literature on problem (1.1) when $f$ is nonsingular. In the semilinear case, i.e. $p=2$, problem (1.1) on a general domain has a long history and has been studied extensively (see e.g. [Am2, Li] and the references therein). The quasilinear case, i.e. $p>1$, has received much attention during the past two decades (see e.g. [GMS, LS1, LS2]). In the case when $f$ is nonsingular and $p$-superlinear at $\infty$, i.e., $\lim _{u \rightarrow \infty} f(u) / u^{p-1}=\infty$, such problems have been investigated in [ANZ, Am1, AAB, DLN, GS, SW] for $p=2$, and in [AAP, DMS, DSS, GMS, HS, HSS] for $p>1$. We are motivated here by the results in [AAP, GMS, HS] concerning the existence of positive solutions to (1.2) when $f$ is $p$-superlinear, $p>1$. In [AAP, Theorem 4.6], assuming that $f \in C^{1}[0, \infty), f(0)<0$, and there exist constants $\beta>0$ and $\alpha \in\left(p, p^{*}\right)$, where $p^{*}=N p / \max (N-p, 0)$, such that

$$
\lim _{u \rightarrow \infty} \frac{f(u)}{u^{\alpha-1}}=\beta
$$

the authors showed that (1.2) has a positive solution for $\lambda>0$ small and there exists a connected set of positive solutions of (1.1) bifurcating from infinity at $\lambda=0$. The result in $[\mathrm{AAP}]$ was extended in [HS, Theorems 2.1, 2.2] to include more general nonlinearities and to cover the case when $f(0)>0$. We refer to [GMS] for related results in the case when $f(0)=0$.

Problems of the type (1.1) with $p=2$ and $f(u)$ singular at $u=0$ arise in the theory of heat conduction in electrical conducting materials, as discussed in [FM]. The model example of this case is

$$
\begin{cases}-\Delta u=A / u^{\alpha}+\gamma u^{q} & \text { in } B, \\ u=0 & \text { on } \partial B\end{cases}
$$

where $A, \gamma, \alpha, q$ are nonnegative constants with $\alpha \in(0,1), q>0, A \neq 0$. Note that when $\gamma \neq 0$, this problem can be reduced to (1.1) with $f(u)=A u^{-\alpha}+u^{p}$ and $\lambda=\gamma^{(1+\alpha) /(q+\alpha)}$ via the transformation $v=\gamma^{1 /(q+\alpha)} u$.

When $A<0$ and $q<1$, the existence of a positive solution to (1.3) for $\gamma$ large was established in [SY, Zh]. The case when $A>0$ was discussed in [CRT, FM, LM] for $\gamma=0$, and in [SY, St] for $\gamma>0$ and $p \in(0,1)$. For $A>0, \gamma>0$ and $q \geq 1$, it was established in $[\mathrm{CP}]$ that there exists a constant $\tilde{\lambda}>0$ such that (1.3) has a positive solution for $\lambda<\tilde{\lambda}$ and no solution for $\lambda>\tilde{\lambda}$. The case when $f(u)$ is bounded away from 0 and $\lim _{u \rightarrow \infty} f(u) / u^{q} \in(0, \infty)$ for some $q \in\left(1,2^{*}\right)$, was considered in $[\mathrm{HKS}]$, in which the authors showed the existence of a constant $\tilde{\lambda}>0$ such that (1.1) with $p=2$ has at least two positive radial solutions for $\lambda<\tilde{\lambda}$, at least one for $\lambda=\tilde{\lambda}$, and none for $\lambda>\tilde{\lambda}$.

In this paper, we are interested in positive radial solutions of the problem (1.1) for $p \geq 2$ when $f$ is $p$-superlinear at $\infty$ and is allowed to be singular at 0 . We 
shall consider both cases when $\lim _{u \rightarrow 0^{+}} f(u)>0$ and $\lim _{u \rightarrow 0^{+}} f(u)<0$. Problems of this kind appear in the the study of chemical reactions, thin films, and nonNewtonian fluids [AA, Di, DHM, DMO, HM]. Our results provide extensions of the results in [AAP, HS] to the singular case, and the results in [HKS] to the case $p \geq 2$ with more general nonlinearities $f(u)$. In particular, the existence result in Theorem 2.1 below deals with the situation when $f$ is $p$-superlinear at $\infty$ and $\lim _{u \rightarrow 0^{+}} f(u)=-\infty$, which occurs in some chemical reactions (see [Di, DHM, $\mathrm{DMO}]$ ) and has not been considered in the literature to our knowledge.

To be more precise, we shall prove in the case $\lim _{u \rightarrow 0^{+}} f(u)<0$ that problem (1.2) has a positive, decreasing solution $u_{\lambda}$ for $\lambda$ small, and $u_{\lambda} \rightarrow \infty$ uniformly on compact subsets of $[0,1)$ as $\lambda \rightarrow 0$.

In the case $\lim _{u \rightarrow 0^{+}} f(u)>0$, we show the existence of a positive number $\lambda^{*}$ such that (1.2) has at least two positive solutions for $\lambda<\lambda^{*}$, at least one for $\lambda=\lambda^{*}$, and none for $\lambda>\lambda^{*}$.

In particular, our results when applied to the model cases

$$
\begin{cases}-\Delta_{p} u=\lambda\left(-1 / u^{\alpha}+u^{q}(\ln (1+u))^{r}\right) & \text { in } B, \\ u=0 & \text { on } \partial B\end{cases}
$$

and

$$
\begin{cases}-\Delta_{p} u=\lambda\left(1 / u^{\alpha}+u^{q}(\ln (1+u))^{r}\right) & \text { in } B, \\ u=0 & \text { on } \partial B,\end{cases}
$$

where $\alpha \in[0,1), r \geq 0, q \in\left(p-1, p^{*}-1\right)$, give the existence of a positive radial solution to (1.4) for $\lambda$ small, and the existence of a constant $\lambda^{*}>0$ such that (1.5) has at least two positive radial solutions for $\lambda<\lambda^{*}$, at least one for $\lambda=\lambda^{*}$, and none for $\lambda>\lambda^{*}$.

Our proofs depend on degree theory and sup- and supersolutions approach as in [HS]. However, the proofs in [HS] do not carry over to the singular case since the compact operator introduced in [HS] is not defined on $C[0,1]$ in that case. To overcome this, we come up with a modified problem whose solutions are fixed points of a compact operator in $C[0,1]$ and then show that these solutions are in fact positive solutions of the original problem.

\section{§2. Main results}

We shall make the following assumptions:

(A.1) $f:(0, \infty) \rightarrow \mathbb{R}$ is continuous and

$$
\lim _{x \rightarrow \infty} \frac{f(x)}{x^{p-1}}=\infty .
$$


(A.2) $N \liminf _{x \rightarrow \infty} \frac{F(x)}{x f(x)}>\max \left(\frac{N}{p}-1,0\right)$, where $F(x)=\int_{0}^{x} f(t) d t$.

(A.3) There exists a constant $\alpha \in[0,1)$ such that

$$
\limsup _{x \rightarrow 0^{+}} x^{\alpha}|f(x)|<\infty \text {. }
$$

(A.4) $f>0$ on $(0, \infty)$ and there exist constants $B>0$ and $\beta \in[0,1)$ such that

$$
\lim _{x \rightarrow 0^{+}} x^{\beta} f(x)=B
$$

By a positive solution of (1.2), we mean a function $u \in C^{1}[0,1]$ with $u>0$ on $[0,1)$ that satisfies (1.2).

Our main results are:

Theorem 2.1. Let (A.1)-(A.3) hold. Then there exists a constant $\lambda_{0}>0$ such that (1.2) has a positive, decreasing solution $u_{\lambda}$ for $\lambda \in\left(0, \lambda_{0}\right)$ with $\left\|u_{\lambda}\right\|_{\infty} \rightarrow \infty$ as $\lambda \rightarrow 0$. Furthermore, there exists a function $L: \mathbb{R}^{+} \rightarrow \mathbb{R}$ with $\lim _{d \rightarrow \infty} L(d)=\infty$ such that

$$
u_{\lambda}(r) \geq L\left(\left\|u_{\lambda}\right\|_{\infty}\right)(1-r) \quad \text { for } r \in[0,1)
$$

Theorem 2.2. Let (A.1)-(A.4) hold. Then there exists a positive constant $\lambda^{*}$ such that (1.2) has at least two positive solutions for $\lambda \in\left(0, \lambda^{*}\right)$, at least one for $\lambda=\lambda^{*}$, and none for $\lambda>\lambda^{*}$.

Remark 2.3. (i) Theorems 2.1 and 2.2 extend Theorems 2.1 and 3.1 of [HS], and Theorem 4.6 of [AAP], to the singular case. Theorem 2.2 with $p=2$ extends Theorem 1 of $[\mathrm{HKS}]$ to nonlinearities $f(u)$ that do not behave like $u^{q}$ at $\infty$.

(ii) When $f$ is nonsingular, condition (A.2) is satisfied under the following assumption introduced in [GMS]:

$(\mathrm{A} .2)^{\prime}$ There exists a constant $\theta \in(0,1)$ such that

$$
N \liminf _{x \rightarrow \infty} \frac{F(\theta x)}{x f_{s}(x)}>\max \left(\frac{N}{p}-1,0\right), \quad \text { where } f_{s}(x)=\sup _{0 \leq t \leq x} f(t) .
$$

It was shown in $[\mathrm{GMS}]$ that when $f$ is nondecreasing, $(\mathrm{A} .2)^{\prime}$ is equivalent to the following condition given in $[\mathrm{TH}]$ :

(A) There exists a constant $\theta \in(0,1)$ such that

$$
N F(\theta x)-\frac{N-p}{p} x f(x) \geq 0 \quad \text { for } x \text { large. }
$$




\section{$\S 3$. Preliminary results}

Let $\psi(r)=1-r$. The following lemma is an extension of Lemma 2.2 of [HS] to the singular case.

Lemma 3.1. Let $\zeta$ be a nonnegative number and let $u$ be the solution of

$$
\left\{\begin{array}{l}
-\left(r^{N-1} \phi\left(u^{\prime}\right)\right)^{\prime}=\lambda r^{N-1} k(r), \quad 0<r<1, \\
u^{\prime}(0)=0, \quad u(1)=\zeta
\end{array}\right.
$$

where $k \geq-m \psi^{-\alpha}$ on $(0,1)$ for some constants $m>0, \alpha \in(0,1)$. Then

(i) $u^{\prime} \leq \phi^{-1}\left(\lambda m_{1}\right)$,

(ii) $u(t) \geq u(s)-\phi^{-1}\left(\lambda m_{1}\right)$ for $0 \leq t \leq s \leq 1$,

(iii) $t^{N-1} \phi\left(u^{\prime}(t)\right) \geq s^{N-1} \phi\left(u^{\prime}(s)\right)-\lambda m_{1}$ for $0 \leq t \leq s \leq 1$, where $m_{1}=$ $m(1-\alpha)^{-1}$.

Proof. Let $u$ be a solution of (3.1). By integrating, we obtain

$$
\begin{aligned}
u^{\prime}(r) & =-\phi^{-1}\left(\frac{\lambda}{r^{N-1}} \int_{0}^{r} \tau^{N-1} k(\tau) d \tau\right) \leq \phi^{-1}\left(\frac{\lambda m}{r^{N-1}} \int_{0}^{r} \tau^{N-1} \psi^{-\alpha} d \tau\right) \\
& \leq \phi^{-1}\left(\lambda m \int_{0}^{r} \psi^{-\alpha} d \tau\right) \leq \phi^{-1}\left(\lambda m_{1}\right)
\end{aligned}
$$

for $r \in(0,1)$, i.e. (i) holds. Integrating this inequality on $(t, s), t<s$, gives

$$
u(s)-u(t) \leq \phi^{-1}\left(\lambda m_{1}\right)(s-t),
$$

which implies (ii). Finally, integrating the equation in (3.1) on $(t, s)$, we obtain (iii).

Lemma $3.2([\mathrm{HW}])$. Let $q>1$. Then there exists a constant $\nu \in(0,1)$ such that for each $g \in L^{q}(0,1)$, the problem

$$
\left\{\begin{array}{l}
-\left(r^{N-1} \phi\left(u^{\prime}\right)\right)^{\prime}=r^{N-1} g, \quad 0<r<1 \\
u^{\prime}(0)=0, \quad u(1)=0
\end{array}\right.
$$

has a unique solution $u \equiv T g \in C^{1, \nu}[0,1]$. Furthermore, there exists a constant $C>0$ independent of $g$ such that

$$
|u|_{1, \nu} \leq C\|g\|_{q}^{1 /(p-1)},
$$

and the operator $T: L^{q}(0,1) \rightarrow C^{1}[0,1]$ is compact. 
Define

$$
\begin{aligned}
& g(x)= \begin{cases}f(x) & \text { if } 0<x \leq 1, \\
f(1) & \text { if } x>1,\end{cases} \\
& h(x)= \begin{cases}0 & \text { if } 0<x \leq 1, \\
f(x)-f(1) & \text { if } x>1,\end{cases}
\end{aligned}
$$

and $h(x)=0$ if $x \leq 0$. Then $h$ is continuous, bounded below on $\mathbb{R}$ and $f=g+h$ on $(0, \infty)$. Using (A.2), it is easily seen that

$$
N \liminf _{x \rightarrow \infty} \frac{H(x)}{x h(x)}>\max \left(\frac{N}{p}-1,0\right)
$$

where $H(x)=\int_{0}^{x} h(t) d t$.

Lemma 3.3. (i) There exist positive constants $C, C_{1}, a, \delta$ with

$$
N / p>a>N / p-1
$$

such that

$$
C H(x)^{a / N} \leq x, \quad h(x) \leq C_{1} H(x)^{1-a / N}
$$

and

$$
N H(x)-\operatorname{axh}(x) \geq \delta H(x)
$$

for $x \gg 1$

(ii) For each $\theta \in(0,1)$, there exists a constant $b_{\theta}$ such that

$$
H(\theta x) \geq b_{\theta} H(x)
$$

for $x \gg 1$. Furthermore, $b_{\theta} \rightarrow 1$ as $\theta \rightarrow 1$.

Proof. In view of (3.4), there exist positive constants $a, \tilde{a}$ such that

$$
N \liminf _{x \rightarrow \infty} \frac{H(x)}{x h(x)}>\tilde{a}>a>\max \left(\frac{N}{p}-1,0\right) .
$$

Hence

$$
H(x) \geq \frac{\tilde{a}}{N} x h(x) \quad \text { for } x \gg 1
$$

which implies

$$
N H(x)-\operatorname{axh}(x) \geq N\left(1-\frac{a}{\tilde{a}}\right) H(x)
$$

and

$$
H^{\prime}(x) \leq \frac{N}{a x} H(x)
$$


for $x \gg 1$. Solving this differential inequality gives

$$
H(x) \leq C_{0} x^{N / a} \quad \text { for } x \gg 1
$$

and so $x \geq\left(H(x) / C_{0}\right)^{a / N}$ for $x \gg 1$. Note that $p<N / a$ since $\lim _{x \rightarrow \infty} H(x) / x^{p}$ $=\infty$. Hence

$$
h(x) \leq \frac{N H(x)}{a x} \leq C_{1} H(x)^{1-a / N}
$$

for $x \gg 1$ and (i) follows. Next, fix $\theta \in(0,1)$. By $(3.5)$,

$$
\int_{\theta x}^{x} h(t) d t=\int_{\theta x}^{x} \frac{t h(t)}{t} d t \leq \frac{N}{\theta a x} \int_{\theta x}^{x} H(t) d t \leq \frac{N(1-\theta)}{\theta a} H(x)
$$

for $x \gg 1$, where we have used the fact that $H(x)$ is increasing for large $x$. Hence

$$
H(\theta x)=H(x)-\int_{\theta x}^{x} h(t) d t \geq b_{\theta} H(x)
$$

for $x \gg 1$, where $b_{\theta}=1-\frac{N(1-\theta)}{\theta a}$.

\section{$\S 4$. Abstract setting and a priori estimates}

Let $\lambda>0$. For $v \in C[0,1]$, define $S_{\lambda} v=\lambda(g(\max (v, \psi))+h(v))$, where $g$ and $h$ are defined by (3.2) and (3.3) respectively. By (A.3), there exists a constant $c_{0}>0$ such that

In particular,

$$
|g(x)| \leq \frac{c_{0}}{x^{\alpha}}+|f(1)| \quad \text { for all } x>0
$$

$$
|g(\max (v, \psi))| \leq \frac{c_{1}}{\psi^{\alpha}}
$$

where $c_{1}=c_{0}+|f(1)|$. This, together with the Lebesgue Dominated Convergence Theorem, implies that $S_{\lambda}: C[0,1] \rightarrow L^{q}(0,1)$ is continuous and maps bounded sets into bounded sets, where $1<q<1 / \alpha$.

Let $A_{\lambda} v=u$, where $u$ is the solution of

$$
\left\{\begin{array}{l}
-\left(r^{N-1} \phi\left(u^{\prime}\right)\right)^{\prime}=\lambda r^{N-1}(g(\max (v, \psi))+h(v)), \quad 0<r<1 \\
u^{\prime}(0)=0, \quad u(1)=0 .
\end{array}\right.
$$

Since $A_{\lambda}=T \circ S_{\lambda}$, where $T$ is defined in Lemma 3.2, it follows that $A_{\lambda}: C[0,1] \rightarrow$ $C[0,1]$ is a compact operator.

Lemma 4.1. There exists a constant $\bar{\lambda}>0$ such that for each $\lambda \in(0, \bar{\lambda})$, there exists a positive constant $r_{\lambda}$ with $\lim _{\lambda \rightarrow 0} r_{\lambda}=\infty$ such that

$$
u=\theta A_{\lambda} u, \theta \in(0,1) \Rightarrow\|u\|_{\infty} \neq r_{\lambda} .
$$


Proof. Let $u$ satisfy $u=\theta A_{\lambda} u$ for some $\theta \in(0,1)$. Then

$$
u(r)=\theta \int_{r}^{1} \phi^{-1}\left(\frac{\lambda}{s^{N-1}} \int_{0}^{s} \tau^{N-1}(g(\max (u, \psi))+h(u)) d \tau\right) d s
$$

which, together with (4.1), implies

$$
\begin{aligned}
|u(r)| & \leq \int_{r}^{1} \phi^{-1}\left(\frac{\lambda}{s^{N-1}} \int_{0}^{s} \tau^{N-1}\left(\frac{c_{1}}{\psi^{\alpha}}+h_{s}\left(\|u\|_{\infty}\right)\right) d \tau\right) d s \\
& \leq \phi^{-1}\left(\lambda c_{2}+\lambda h_{s}\left(\|u\|_{\infty}\right)\right)
\end{aligned}
$$

for $r \in(0,1)$, where $c_{2}=c_{1}(1-\alpha)^{-1}$ and $h_{s}(t)=\sup _{x \in[0, t]}|h(x)|$.

Hence

$$
\phi\left(\|u\|_{\infty}\right) \leq \lambda\left(c_{2}+h_{s}\left(\|u\|_{\infty}\right)\right)
$$

Let $\bar{\lambda}=\frac{1}{2\left(c_{2}+h_{s}(1)\right)}$ and $\lambda \in(0, \bar{\lambda})$. Then

$$
c_{2}+h_{s}(1)=\frac{1}{2 \bar{\lambda}}<\frac{1}{2 \lambda} .
$$

Since $\lim _{x \rightarrow \infty} \frac{c_{2}+h_{s}(x)}{\phi(x)}=\infty$, there exists a constant $r_{\lambda}>1$ such that

$$
\frac{c_{2}+h_{s}\left(r_{\lambda}\right)}{\phi\left(r_{\lambda}\right)}=\frac{1}{2 \lambda}
$$

Clearly $\lim _{\lambda \rightarrow 0} r_{\lambda}=\infty$, and from (4.3) and (4.4), we see that $\|u\|_{\infty} \neq r_{\lambda}$.

Lemma 4.2. For each $\lambda>0$, there exists a constant $\zeta_{\lambda}>0$ such that

$$
u=A_{\lambda} u+\zeta, \zeta \geq 0 \Rightarrow \zeta \leq \zeta_{\lambda} .
$$

Proof. Let $u$ satisfy $u=A_{\lambda} u+\zeta$, where $\zeta \geq 0$ and $\lambda>0$. Then

$$
\left\{\begin{array}{l}
-\left(r^{N-1}\left|u^{\prime}\right|^{p-2} u^{\prime}\right)^{\prime}=\lambda r^{N-1}(g(\max (u, \psi))+h(u)), \quad 0<r<1, \\
u^{\prime}(0)=0, \quad u(1)=\zeta .
\end{array}\right.
$$

Let $\lambda_{1}>0$ be the first eigenvalue of $-\Delta_{p}$ on the unit ball with Dirichlet boundary conditions, and let $\phi_{1}$ be the corresponding normalized positive radial eigenfunction, i.e. $\left\|\phi_{1}\right\|_{\infty}=1, \phi_{1}>0$ in $[0,1)$, and

$$
\left\{\begin{aligned}
-\left(r^{N-1}\left|\phi_{1}^{\prime}\right|^{p-2} \phi_{1}^{\prime}\right)^{\prime} & =\lambda_{1} r^{N-1} \phi_{1}^{p-1}, \quad 0<r<1, \\
\phi_{1}^{\prime}(0)=0, \quad \phi_{1}(1) & =0 .
\end{aligned}\right.
$$

Since there exists a constant $m>0$ such that

$$
g(\max (v, \psi))+h(v) \geq-\frac{c_{1}}{\psi^{\alpha}}+h(v) \geq-\frac{m}{\psi^{\alpha}}
$$


for all $v \in C[0,1]$, Lemma 3.1(ii) implies

$$
u(r) \geq \zeta-\phi^{-1}\left(\lambda m_{1}\right), \quad \text { where } \quad m_{1}=m(1-\alpha)^{-1} .
$$

Choose $\zeta_{\lambda}$ so that $\zeta_{\lambda}>\max \left\{2 \phi^{-1}\left(\lambda m_{1}\right), 2\right\}$ and

$$
\frac{f(x)}{x^{p-1}}>\frac{2 \lambda_{1}}{\lambda} \quad \text { for } x>\frac{\zeta_{\lambda}}{2} \text {. }
$$

We claim that $\zeta \leq \zeta_{\lambda}$. Suppose $\zeta>\zeta_{\lambda}$ and let $\tilde{u}=u-\zeta$.

Since

$$
u(r) \geq \zeta_{\lambda} / 2>\psi \quad \text { for } r \in(0,1),
$$

it follows that

$$
\left\{\begin{array}{l}
-\left(r^{N-1}\left|\tilde{u}^{\prime}\right|^{p-2} \tilde{u}^{\prime}\right)^{\prime}=\lambda r^{N-1} f(u) \geq 2 \lambda_{1} r^{N-1}(\tilde{u}+\zeta)^{p-1} \quad \text { in }(0,1), \\
\tilde{u}^{\prime}(0)=0, \quad \tilde{u}(1)=0 .
\end{array}\right.
$$

By the strong maximum principle, $\tilde{u}>0$ in $[0,1)$ and $\tilde{u}^{\prime}(1)<0$. Let $c$ be largest such that $\tilde{u} \geq c \phi_{1}$ in $[0,1)$. Then $c>0$ and

$$
-\left(r^{N-1}\left|\tilde{u}^{\prime}\right|^{p-2} \tilde{u}^{\prime}\right)^{\prime} \geq 2 \lambda_{1} r^{N-1}\left(c \phi_{1}\right)^{p-1} \quad \text { in }(0,1),
$$

and the weak comparison principle implies $\tilde{u} \geq 2^{1 /(p-1)} c \phi_{1}$ in $[0,1)$, a contradiction with the choice of $c$. Thus $\zeta \leq \zeta_{\lambda}$, as claimed.

Lemma 4.3. Let $\lambda<\bar{\lambda}$ and let $u$ satisfy

$$
u=A_{\lambda} u+\zeta
$$

for some $\zeta \geq 0$. Then there exists a positive constant $C_{\bar{\lambda}}$ such that

$$
\|u\|_{\infty}=u(0) \quad \text { whenever }\|u\|_{\infty}>C_{\bar{\lambda}} .
$$

Proof. Suppose $\|u\|_{\infty} \equiv d=\left|u\left(r_{1}\right)\right|$ for some $r_{1} \in(0,1)$. By Lemma 3.1(ii),

$$
u\left(r_{1}\right) \geq-\phi^{-1}\left(\lambda m_{1}\right),
$$

and so $u\left(r_{1}\right)>0$ if $d>2 \phi^{-1}\left(\bar{\lambda} m_{1}\right)$. For such $d$,

$$
u(r) \geq u\left(r_{1}\right)-\phi^{-1}\left(\bar{\lambda} m_{1}\right) \geq d / 2
$$

for $r \in\left(0, r_{1}\right)$. By integrating and using (4.1), we obtain

$$
\begin{aligned}
-u^{\prime}(r) & =\phi^{-1}\left(\frac{\lambda}{r^{N-1}}\left(\int_{0}^{r} \tau^{N-1}(g(\max (u, \psi))+h(u)) d \tau\right)\right. \\
& \geq \phi^{-1}\left(\frac{\lambda}{r^{N-1}} \int_{0}^{r} \tau^{N-1}\left(-\frac{c_{1}}{\psi^{\alpha}}+h(u)\right) d \tau\right) \\
& \geq \phi^{-1}\left(\lambda r\left\{-\frac{c_{1}}{1-\alpha}+\frac{1}{N} h_{i}\left(\frac{d}{2}\right)\right\}\right)>0
\end{aligned}
$$


for $r \in\left(0, r_{1}\right)$, where $h_{i}(t)=\inf _{x \geq t} h(x)$, provided that $d \gg 1$. Here we have used the fact that

$$
r^{-N} \int_{0}^{r} \frac{\tau^{N-1}}{(1-\tau)^{\alpha}} d \tau \leq(1-\alpha)^{-1}
$$

for $r \in(0,1)$, and $h_{i}(t) \rightarrow \infty$ as $t \rightarrow \infty$. Thus $u$ is decreasing on $\left(0, r_{1}\right)$ and so $u(0)>u\left(r_{1}\right)$, a contradiction.

Lemma 4.4. Let $\lambda<\bar{\lambda}$ and $\zeta_{0}>0$. Suppose $u$ satisfies

$$
u=A_{\lambda} u+\zeta
$$

for some $0 \leq \zeta \leq \zeta_{0}$. Then:

(i) There exists a function $L: \mathbb{R}^{+} \rightarrow \mathbb{R}$ depending on $\zeta_{0}$ and $\bar{\lambda}$ with $\lim _{d \rightarrow \infty} L(d)$ $=\infty$ such that

$$
u(r) \geq L\left(\|u\|_{\infty}\right)(1-r) \quad \text { for } r \in(0,1) .
$$

(ii) There exists a constant $\bar{R}>0$ depending on $\zeta_{0}$ and $\bar{\lambda}$ such that $u$ is decreasing on $(0,1)$ if $\|u\|_{\infty}>\bar{R}$.

(iii) If $\lambda>\underline{\lambda}>0$ then there exists a constant $R>0$ depending on $\underline{\lambda}, \bar{\lambda}, \zeta_{0}$ such that $\|u\|_{\infty}<R$.

Proof. Note that

$$
\left\{\begin{array}{l}
-\left(r^{N-1}\left|u^{\prime}\right|^{p-2} u^{\prime}\right)^{\prime}=\lambda r^{N-1}(g(\max (u, \psi))+h(u)), \quad 0<r<1 \\
u^{\prime}(0)=0, \quad u(1)=\zeta
\end{array}\right.
$$

Multiplying the equation in (4.5) by $r u^{\prime}$ gives

$$
\begin{aligned}
\left(r^{N}\left(1-\frac{1}{p}\right)\left|u^{\prime}\right|^{p}+\lambda r^{N} H(u)\right)^{\prime} & =-\lambda r^{N} g(\max (u, \psi)) u^{\prime} \\
& +\lambda r^{N-1} N H(u)+r^{N-1}\left(1-\frac{N}{p}\right)\left|u^{\prime}\right|^{p} .
\end{aligned}
$$

Next, multiplying the equation in (4.5) by $a$, where $a$ is given by Lemma 3.3(i), we obtain

$$
\left(a r^{N-1}\left|u^{\prime}\right|^{p-2} u^{\prime} u\right)^{\prime}=-\lambda a r^{N-1} g(\max (u, \psi)) u-\lambda r^{N-1} a u h(u)+r^{N-1} a\left|u^{\prime}\right|^{p} .
$$

Adding (4.6) and (4.7) yields

$$
\begin{aligned}
\psi^{\prime}(r)= & r^{N-1}\left(a+1-\frac{N}{p}\right)\left|u^{\prime}\right|^{p}+\lambda r^{N-1}(N H(u)-a u h(u)) \\
& -\lambda r^{N} g(\max (u, \psi)) u^{\prime}-\lambda a r^{N-1} g(\max (u, \psi)) u,
\end{aligned}
$$

where $\psi(r)=r^{N}(1-1 / p)\left|u^{\prime}\right|^{p}+\lambda r^{N} H(u)+a r^{N-1}\left|u^{\prime}\right|^{p-2} u^{\prime} u$. 
In what follows, we shall denote by $K_{i}, i=0,1, \ldots$, positive constants independent of $u$.

By Lemma 3.3(i), there exist constants $\delta, K_{0}>0$ such that

$$
N H(x)-\operatorname{axh}(x) \geq \delta H(x)-K_{0}
$$

for all $x \in \mathbb{R}$. Hence

$$
\begin{gathered}
\psi^{\prime}(r) \geq \lambda \delta r^{N-1} H(u)-\lambda r^{N} g(\max (u, \psi)) u^{\prime} \\
-\lambda a r^{N-1} g(\max (u, \psi)) u-\lambda K_{0}
\end{gathered}
$$

for $r \in(0,1)$. In view of Lemma 3.3(ii), there exists $\theta \in(0,1)$ such that

$$
H(\theta x) \geq(1 / 2) H(x) \quad \text { for } x \gg 1 \text {. }
$$

Suppose $\|u\|_{\infty}=d \gg 1$. Then Lemma 4.3 implies $\|u\|_{\infty}=u(0)$. Let $\bar{\theta} \in(\theta, 1)$ and $r_{0} \in(0,1)$ be such that $u\left(r_{0}\right)=\bar{\theta} d$. Note that $r_{0}$ exists since $u(0)>\bar{\theta} d$ and $u(1)=\zeta \leq \zeta_{0}<\bar{\theta} d$ for large $d$.

By Lemma 3.1(ii),

$$
u(r) \geq u\left(r_{0}\right)-\phi^{-1}\left(\bar{\lambda} m_{1}\right) \geq \theta d
$$

for $r<r_{0}$. From (4.11) and (4.12), for $r>r_{0}$ we obtain

$$
\begin{aligned}
\lambda \delta \int_{0}^{r} s^{N-1} H(u) d s & \geq \lambda \delta \int_{0}^{r_{0}} s^{N-1} H(u) d s-\lambda K_{1} \\
& \geq \frac{\lambda \delta r_{0}^{N}}{N} H(\theta d)-\lambda K_{1} \\
& \geq \frac{\lambda \delta r_{0}^{N}}{2 N} H(d)-\lambda K_{1} .
\end{aligned}
$$

Integrating (4.10) on $(0, r)$, where $r \in\left(r_{0}, 1\right)$, and using (4.13), we obtain

$$
\begin{aligned}
\psi(r) \geq & \frac{\lambda \delta r_{0}^{N}}{2 N} H(d)-\lambda \int_{0}^{r} s^{N} g(\max (u, \psi)) u^{\prime} d s \\
& -\lambda a \int_{0}^{r} s^{N-1} g(\max (u, \psi)) u d s-\lambda K_{1} .
\end{aligned}
$$

Since $p \geq 2$, it follows from Lemma 3.1(i) that there exists a positive constant $C_{0}$ depending on $\bar{\lambda}$ such that

$$
u^{\prime} \geq \phi\left(u^{\prime}\right)-C_{0}
$$

in $(0,1)$, which together with (4.1) implies 


$$
\begin{aligned}
& -\lambda \int_{0}^{r} s^{N} g(\max (u, \psi)) u^{\prime} d s \\
= & -\lambda \int_{0}^{r} s^{N}\left(g(\max (u, \psi))+\frac{c_{1}}{\psi^{\alpha}}\right)\left(u^{\prime}-\phi^{-1}\left(\lambda m_{1}\right)\right) d s+\lambda c_{1} \int_{0}^{r} \frac{s^{N} u^{\prime}}{\psi^{\alpha}} d s \\
& -\lambda \phi^{-1}\left(\lambda m_{1}\right) \int_{0}^{r} s^{N}\left(g(\max (u, \psi))+\frac{c_{1}}{\psi^{\alpha}}\right) d s \\
\geq & \lambda c_{1} \int_{0}^{r} \frac{s^{N}\left(\phi\left(u^{\prime}\right)-C_{0}\right)}{\psi^{\alpha}} d s-\lambda \phi^{-1}\left(\lambda m_{1}\right) \int_{0}^{r} s^{N}\left(g(\max (u, \psi))+\frac{c_{1}}{\psi^{\alpha}}\right) d s \\
\geq & \lambda c_{1} \int_{0}^{r} \frac{s^{N} \phi\left(u^{\prime}\right)}{\psi^{\alpha}} d s-K_{2} .
\end{aligned}
$$

By Lemma 3.1(iii),

$$
\int_{0}^{r} \frac{s^{N} \phi\left(u^{\prime}\right)}{\psi^{\alpha}} d s \geq\left(r^{N-1} \phi\left(u^{\prime}(r)\right)-\lambda m_{1}\right)\left(\int_{0}^{r} \frac{s}{\psi^{\alpha}} d s\right) .
$$

From this and (4.16), we get

$$
-\lambda \int_{0}^{r} s^{N} g(\max (u, \psi)) u^{\prime} d s \geq \lambda c_{1}\left(\int_{0}^{r} \frac{s}{\psi^{\alpha}} d s\right) r^{N-1} \phi\left(u^{\prime}(r)\right)-K_{3} .
$$

Next, using Lemma 3.1(ii), (4.1), and integration by parts, we obtain

$$
\begin{aligned}
& -\lambda a \int_{0}^{r} s^{N-1} g(\max (u, \psi)) u d s \\
= & -\lambda a \int_{0}^{r} s^{N-1}\left(g(\max (u, \psi))+\frac{c_{1}}{\psi^{\alpha}}\right)\left(u+\phi^{-1}\left(\lambda m_{1}\right)\right) d s \\
& +\lambda a c_{1} \int_{0}^{r} \frac{s^{N-1} u}{\psi^{\alpha}} d s+\lambda a \phi^{-1}\left(\lambda m_{1}\right) \int_{0}^{r} s^{N-1}\left(g(\max (u, \psi))+\frac{c_{1}}{\psi^{\alpha}}\right) d s \\
\geq & -\lambda a c_{1} \int_{0}^{r} \frac{s^{N-1} u}{\psi^{\alpha}} d s-K_{4} \\
= & -\lambda a c_{1}\left(\int_{0}^{r} \frac{s^{N-1}}{\psi^{\alpha}} d \tau\right) u(r)+\lambda a c_{1} \int_{0}^{r}\left(\int_{0}^{s} \frac{\tau^{N-1}}{\psi^{\alpha}} d \tau\right) u^{\prime} d s-K_{4} .
\end{aligned}
$$

From (4.15) and Lemma 3.1(i) \& (iii),

$$
\begin{aligned}
& \text { (4.19) } \quad \int_{0}^{r}\left(\int_{0}^{s} \frac{\tau^{N-1}}{\psi^{\alpha}} d \tau\right) u^{\prime} d s \\
& =\int_{0}^{r}\left(\int_{0}^{s} \frac{\tau^{N-1}}{\psi^{\alpha}} d \tau\right)\left(u^{\prime}-\phi^{-1}\left(\lambda m_{1}\right)\right) d s+\phi^{-1}\left(\lambda m_{1}\right) \int_{0}^{r}\left(\int_{0}^{s} \frac{\tau^{N-1}}{\psi^{\alpha}} d \tau\right) d s \\
& \geq \int_{0}^{r}\left(\int_{0}^{s} \frac{d \tau}{\psi^{\alpha}}\right) s^{N-1}\left(u^{\prime}-\phi^{-1}\left(\lambda m_{1}\right)\right) d s \geq r^{N-1} \phi\left(u^{\prime}(r)\right) \int_{0}^{r}\left(\int_{0}^{s} \frac{d \tau}{\psi^{\alpha}}\right) d s-K_{5} .
\end{aligned}
$$


Combining (4.18) and (4.19) gives

$$
\begin{aligned}
-\lambda a \int_{0}^{r} s^{N-1} g(\max (u, \psi)) u d s & \geq-\lambda a c_{1}\left(\int_{0}^{r} \frac{s^{N-1}}{\psi^{\alpha}} d s\right) u(r) \\
+\lambda a c_{1} & \left(\int_{0}^{r}\left(\int_{0}^{s} \frac{d \tau}{\psi^{\alpha}}\right) d s\right) r^{N-1} \phi\left(u^{\prime}(r)\right)-K_{6} .
\end{aligned}
$$

We shall need an estimate on $r_{0}$. By Lemma 3.3(i),

$$
\begin{aligned}
-u^{\prime}(r) & =\phi^{-1}\left(\frac{\lambda}{r^{N-1}}\left(\int_{0}^{r} \tau^{N-1}(g(\max (u, \psi))+h(u)) d \tau\right)\right. \\
& \leq \phi^{-1}\left(\frac{\lambda}{r^{N-1}}\left(\int_{0}^{r} \tau^{N-1}\left(\frac{c_{1}}{\psi^{\alpha}}+C_{1} H(u)^{1-a / N}+K_{7}\right) d \tau\right)\right) \\
& \left.\leq\left(2 \lambda C_{1} H(d)^{1-a / N} r\right)\right)^{1 /(p-1)}
\end{aligned}
$$

for $r \in(0,1)$. Integrating this inequality on $\left(0, r_{0}\right)$ and using Lemma 3.3(i), we get $C(1-\bar{\theta}) H(d)^{a / N} \leq(1-\bar{\theta}) d \leq((p-1) / p)\left(2 \lambda C_{1}\right)^{1 /(p-1)} H(d)^{(1-a / N) 1 /(p-1)} r_{0}^{p /(p-1)}$, which implies

$$
r_{0} \geq \frac{K_{8}}{\lambda^{1 / p}} H(d)^{a / N-1 / p} .
$$

Next, integrating $(4.21)$ on $(0,1)$ gives

$$
d \leq \zeta_{0}+K_{9} \lambda^{1 /(p-1)} H(d)^{(1-a / N) /(p-1)},
$$

and therefore, if $d \geq 2 \zeta_{0}$,

$$
C H(d)^{a / N} \leq d \leq 2 K_{9} \lambda^{1 /(p-1)} H(d)^{(1-a / N) /(p-1)},
$$

which implies

$$
\lambda \geq K_{10} H(d)^{a p / N-1} .
$$

If $N \geq p$ then it follows from (4.22) that

$$
\lambda r_{0}^{N} H(d) \geq \lambda^{1-N / p} K_{8}^{N} H(d)^{a+1-N / p} \geq \bar{\lambda}^{1-N / p} K_{8}^{N} H(d)^{a+1-N / p},
$$

while if $N<p$, we deduce from (4.22) and (4.23) that

$$
\begin{aligned}
\lambda r_{0}^{N} H(d) & \geq \lambda^{1-N / p} K_{8}^{N} H(d)^{a+1-N / p} \\
& \geq\left(K_{10} H(d)^{a p / N-1}\right)^{1-N / p} K_{8}^{N} H(d)^{a+1-N / p} \\
& =K_{11} H(d)^{a p / N} .
\end{aligned}
$$


Combining (4.14), (4.17), (4.20), (4.24), and (4.25), we get

$$
\begin{aligned}
\psi(r) \geq & K_{12} H_{1}(d)+\lambda c_{1}\left(\int_{0}^{r} \frac{s}{\psi^{\alpha}} d s\right) r^{N-1} \phi\left(u^{\prime}(r)\right)-\lambda a c_{1}\left(\int_{0}^{r} \frac{s^{N-1}}{\psi^{\alpha}} d s\right) u(r) \\
& +\lambda a c_{1}\left(\int_{0}^{r}\left(\int_{0}^{s} \frac{d \tau}{\psi^{\alpha}}\right) d s\right) r^{N-1} \phi\left(u^{\prime}(r)\right)-K_{13}
\end{aligned}
$$

for $r \in\left(r_{0}, 1\right)$, where $H_{1}(d)=H(d)^{\gamma}, \gamma=a+1-N / p$ if $N \geq p$, and $\gamma=a p / N$ if $N<p$.

Let $k>0$ be such that $\tilde{H}(x) \equiv H(x)+k x$ is increasing on $\mathbb{R}$. Since we have $\lim _{x \rightarrow \infty} H(x) / x^{p}=\infty$, there exist constants $k_{1}$ and $K_{14}$ such that

$$
\begin{aligned}
\psi(r)-\lambda c_{1} & \left(\int_{0}^{r} \frac{s}{\psi^{\alpha}} d s\right) r^{N-1} \phi\left(u^{\prime}(r)\right)+\lambda a c_{1}\left(\int_{0}^{r} \frac{s^{N-1}}{\psi^{\alpha}} d s\right) u(r) \\
& -\lambda a c_{1}\left(\int_{0}^{r}\left(\int_{0}^{s} \frac{d \tau}{\psi^{\alpha}}\right) d s\right) r^{N-1} \phi\left(u^{\prime}(r)\right) \leq k_{1} \tilde{H}\left(|u(r)|+\left|u^{\prime}(r)\right|\right)+K_{14}
\end{aligned}
$$

for $r \in\left(r_{0}, 1\right)$. Consequently,

$$
|u(r)|+\left|u^{\prime}(r)\right| \geq \tilde{H}^{-1}\left(\frac{K_{12} H_{1}(d)-K_{14}}{k_{1}}\right) .
$$

By Lemma 3.1,

$$
|u|+\left|u^{\prime}\right| \leq u-u^{\prime}+4 \phi^{-1}\left(\bar{\lambda} m_{1}\right)
$$

and so

$$
-u^{\prime}+u \geq H_{2}(d) \quad \text { on }\left(r_{0}, 1\right)
$$

where $H_{2}(d)=\tilde{H}^{-1}\left(\frac{K_{12} H_{1}(d)-K_{14}}{k_{1}}\right)-4 \phi^{-1}\left(\bar{\lambda} m_{1}\right)$. Note that $H_{2}(d) \rightarrow \infty$ as $d \rightarrow \infty$. Solving the above differential inequality, we get

$$
u(r) \geq e^{r-1} \zeta+e^{r}\left(\int_{r}^{1} e^{-s} d s\right) H_{2}(d) \geq \frac{H_{2}(d)}{e}(1-r)
$$

for $r>r_{0}$ and $d \gg 1$, while (4.12) holds for $r \leq r_{0}$ and $d \gg 1$. On the other hand, if $d<d_{0}$ for some $d_{0}>0$ then it follows from the integral formula for $u^{\prime}$ that $\left\|u^{\prime}\right\|_{\infty}<D_{0}$, where $D_{0}$ depends on $d_{0}$ and $\bar{\lambda}$. Hence

$$
u(r)=\zeta-\int_{r}^{1} u^{\prime} \geq-D_{0}(1-r) \quad \text { for } r \in(0,1) .
$$

Hence (i) follows.

(ii) Let $h_{0}$ be a positive constant such that $h(x) \geq-h_{0}$ for all $x \in \mathbb{R}$, and let $\tilde{R}>2 \phi^{-1}\left(\bar{\lambda} m_{1}\right)$ be large enough so that

$$
h_{i}(\tilde{R})>N 2^{N+2}\left(c_{1}(1-\alpha)^{-1}+h_{0}\right),
$$


where $h_{i}(t)=\inf _{x \geq t} h_{i}(x)$, and $c_{1}$ is given by (4.1). Choose $\bar{R}>0$ so that

$$
L(z)>4 \tilde{R} \quad \text { for } z \geq \bar{R} .
$$

Suppose $\|u\|_{\infty}>\bar{R}$. Then, by part (i),

$$
\frac{u(1 / 2)}{2} \geq \frac{1}{4} L\left(\|u\|_{\infty}\right)>\tilde{R}
$$

Since

$$
-\phi\left(u^{\prime}(r)\right) \geq \frac{\lambda}{r^{N-1}} \int_{0}^{r} \tau^{N-1}\left(-\frac{c_{1}}{\psi^{\alpha}}+h(u)\right) d \tau
$$

and

$$
u(\tau) \geq u(1 / 2)-\phi^{-1}\left(\bar{\lambda} m_{1}\right) \geq \frac{u(1 / 2)}{2}
$$

for $\tau \leq 1 / 2$, it follows that

$$
\begin{aligned}
\int_{0}^{r} \tau^{N-1}\left(-\frac{c_{1}}{\psi^{\alpha}}+h(u)\right) d \tau & \geq \frac{r^{N}}{N}\left(h_{i}\left(\frac{u(1 / 2)}{2}\right)-N c_{1}(1-\alpha)^{-1}-h_{0}\right) \\
& \geq \frac{r^{N}}{2 N} h_{i}\left(\frac{u(1 / 2)}{2}\right)>0
\end{aligned}
$$

for $r \leq 1 / 2$. Hence $u^{\prime}<0$ on $(0,1 / 2]$. For $r>1 / 2$,

$$
\begin{aligned}
\int_{0}^{r} \tau^{N-1}\left(-\frac{c_{1}}{\psi^{\alpha}}+h(u)\right) d \tau= & \int_{0}^{1 / 2} \tau^{N-1}\left(-\frac{c_{1}}{\psi^{\alpha}}+h(u)\right) d \tau \\
& +\int_{1 / 2}^{r} \tau^{N-1}\left(-\frac{c_{1}}{\psi^{\alpha}}+h(u)\right) d \tau \\
& \geq \frac{1}{2^{N+1} N} h_{i}\left(\frac{u(1 / 2)}{2}\right)-c_{1}(1-\alpha)-h_{0} \\
& >\frac{1}{2^{N+2} N} h_{i}\left(\frac{u(1 / 2)}{2}\right)
\end{aligned}
$$

and (ii) follows.

(iii) Let $R_{1}>0$ be such that

$$
\frac{h_{i}(x)}{\phi(x)}>\frac{N 2^{N+2 p}}{\bar{\lambda}}
$$

for $x \geq R_{1}$. Let $R>\bar{R}$ be such that

$$
L(z)>4 R_{1} \quad \text { for } z \geq R,
$$

where $\bar{R}$ is defined in part (ii). We claim that $\|u\|_{\infty}<R$. Suppose $\|u\|_{\infty} \geq R$. 
Then, by integrating on $(1 / 2,1)$ the inequality

$$
-u^{\prime} \geq \phi^{-1}\left(\frac{\lambda}{2^{N+2} N} h_{i}\left(\frac{u(1 / 2)}{2}\right)\right),
$$

obtained from (4.26) and (4.27), we get

$$
2 u(1 / 2) \geq \phi^{-1}\left(\frac{\lambda}{2^{N+2} N} h_{i}\left(\frac{u(1 / 2)}{2}\right)\right),
$$

or, equivalently,

$$
\frac{h_{i}\left(\frac{u(1 / 2)}{2}\right)}{\phi\left(\frac{u(1 / 2)}{2}\right)} \leq \frac{N 2^{N+2 p}}{\lambda}<\frac{N 2^{N+2 p}}{\underline{\lambda}} .
$$

This implies $u(1 / 2) / 2<R_{1}$, and since

$$
L\left(\|u\|_{\infty}\right) \leq 2 u(1 / 2)<4 R_{1},
$$

it follows that $\|u\|_{\infty}<R$, a contradiction which proves the claim. This completes the proof of Lemma 4.4 .

\section{$\S 5$. Proofs of the main results}

Proof of Theorem 2.1. Suppose $\lambda<\bar{\lambda}$, where $\bar{\lambda}$ is defined by Lemma 4.1. In view of Lemmas 4.1, 4.2, and 4.4(iii), it follows that

$$
\operatorname{deg}\left(I-A_{\lambda}, B\left(0, r_{\lambda}\right), 0\right)=1, \quad \operatorname{deg}\left(I-A_{\lambda}, B(0, R), 0\right)=0,
$$

and the excision property of the Leray-Schauder degree gives the existence of a fixed point $u_{\lambda}$ of $A_{\lambda}$ such that

$$
\left\|u_{\lambda}\right\|_{\infty}>r_{\lambda}
$$

Since $r_{\lambda} \rightarrow \infty$ as $\lambda \rightarrow 0$, it follows from Lemma 4.4(i) \& (ii) with $\zeta_{0}=0$ that, for $\lambda$ small, $u_{\lambda}$ is decreasing and

$$
u_{\lambda}(r) \geq L\left(\left\|u_{\lambda}\right\|_{\infty}\right)(1-r) \geq \psi(r)
$$

for $r \in[0,1]$. In particular, $u_{\lambda}$ is a positive solution of (1.2) for $\lambda>0$ small and $u_{\lambda} \rightarrow \infty$ uniformly on compact subsets of $[0,1)$. This completes the proof of Theorem 2.1.

We now turn our attention to the positone case. By (A.1) and (A.4), there exists a positive number $\kappa$ such that

$$
f(x) \geq \kappa \quad \text { for all } x>0 .
$$

Let $\psi_{\lambda}=c_{\lambda} \psi$, where $c_{\lambda}=(\lambda \kappa / N)^{1 /(p-1)}(p-1) / p$. 
For $\lambda>0$ and $v \in C[0,1]$, let $u=\tilde{A}_{\lambda} v$ be the solution of

$$
\left\{\begin{array}{l}
\left.-\left(r^{N-1} \phi\left(u^{\prime}\right)\right)^{\prime}=\lambda r^{N-1}\left(g \max \left(v, \psi_{\lambda}\right)\right)+h(v)\right), \quad 0<r<1, \\
u^{\prime}(0)=0, \quad u(1)=0 .
\end{array}\right.
$$

Then $\tilde{A}_{\lambda}: C[0,1] \rightarrow C[0,1]$ is a compact operator and using the same arguments as above, we obtain the following results for $\tilde{A}_{\lambda}$.

Lemma 5.1. (i) Let $0<\underline{\lambda}<\lambda<\bar{\lambda}$. Then there exists a positive number $R_{0}>0$ depending on $\underline{\lambda}$ and $\bar{\lambda}$ such that any solution $u_{\lambda}$ of

$$
u=\tilde{A}_{\lambda} u
$$

satisfies $\|u\|_{\infty}<R_{0}$. Furthermore

$$
\operatorname{deg}\left(I-\tilde{A}_{\lambda}, B\left(0, R_{0}\right), 0\right)=0 .
$$

(ii) $\tilde{A}_{\lambda}$ has a fixed point for $\lambda$ small.

Lemma 5.2. (i) Let u satisfy

$$
\left\{\begin{array}{l}
-\left(r^{N-1} \phi\left(u^{\prime}\right)\right)^{\prime} \geq \lambda r^{N-1} \kappa, \quad 0<r<1 \\
u^{\prime}(0)=0, \quad u(1)=0
\end{array}\right.
$$

Then $u \geq \psi_{\lambda}$ in $(0,1)$. In particular, $u$ is a fixed point of $\tilde{A}_{\lambda}$ if and only if $u$ is a solution of (1.2).

(ii) There exists a positive number $\tilde{\lambda}$ such that (1.2) has no solution for $\lambda \geq \tilde{\lambda}$.

Proof. (i) Using the integral formula for $u$, we see that

$$
\begin{aligned}
u(r) & \geq \int_{r}^{1} \phi^{-1}\left(\frac{\lambda}{s^{N-1}} \int_{0}^{s} \tau^{N-1} \kappa d \tau\right) d s=\int_{r}^{1}(\lambda \kappa s / N)^{1 /(p-1)} d s \\
& \geq(\lambda \kappa / N)^{1 /(p-1)}((p-1) / p)(1-r)
\end{aligned}
$$

for $r \in(0,1)$. Consequently, if $u$ is a solution of (1.2) then $u=\max \left(u, \psi_{\lambda}\right)$ and so $u$ is a fixed point of $\tilde{A}_{\lambda}$. Conversely, suppose $u=\tilde{A}_{\lambda} u$. Since

$$
g\left(\max \left(u, \psi_{\lambda}\right)\right)+h(u)=f\left(\max \left(u, \psi_{\lambda}\right)\right)
$$

if $\max \left(u, \psi_{\lambda}\right) \leq 1$, and

$$
g\left(\max \left(u, \psi_{\lambda}\right)\right)+h(u)=f(\max (u, 1))
$$

if $\max \left(u, \psi_{\lambda}\right)>1$, it follows that $u \geq \psi_{\lambda}$ in $(0,1)$, and so $u$ is a positive solution of $(1.2)$. 
(ii) Let $u$ be a solution of (1.2). Then $u$ is decreasing and satisfies

$$
\begin{aligned}
u(1 / 2) & \geq \int_{1 / 2}^{1} \phi^{-1}\left(\frac{\lambda}{s^{N-1}} \int_{0}^{1 / 2} \tau^{N-1} f(u) d \tau\right) d s \\
& \geq \frac{1}{2} \phi^{-1}\left(\frac{\lambda}{N 2^{N}} f_{i}(u(1 / 2))\right)
\end{aligned}
$$

or

$$
\frac{f_{i}(u(1 / 2)}{\phi(u(1 / 2))} \leq \frac{N 2^{N+p-1}}{\lambda}
$$

which is a contradiction to (5.3) and the fact that $\lim _{x \rightarrow \infty} f_{i}(x) / \phi(x)=\infty$ if $\lambda$ is sufficiently large.

Let $\Lambda=\{\lambda>0:(1.2)$ has a solution $\}$ and let $\lambda^{*}=\sup \Lambda$.

Lemma 5.3. $\lambda^{*} \in(0, \infty)$ and $\lambda^{*} \in \Lambda$.

Proof. Using Lemmas 5.1(ii) and 5.2(ii), we see that $\lambda^{*} \in(0, \infty)$. Let $\left(\lambda_{n}\right)$ be a sequence in $\Lambda$ such that $\lambda_{n} \rightarrow \lambda^{*}$ and let $\left(u_{n}\right)$ be the corresponding solutions of (1.2). By Lemma 5.1(i), $\left(\left\|u_{n}\right\|_{\infty}\right)$ is bounded, and so there exists a constant $C>0$ such that

$$
f\left(u_{n}\right) \leq \frac{C}{u_{n}^{\alpha}} \leq \frac{C}{c_{\lambda_{n}}^{\alpha} \psi^{\alpha}} \quad \text { in }(0,1)
$$

for all $n$. From this and the formula

$$
u_{n}^{\prime}(r)=-\phi^{-1}\left(\frac{\lambda_{n}}{r^{N-1}} \int_{0}^{r} \tau^{N-1} f\left(u_{n}\right) d \tau\right)
$$

we deduce that

$$
\left|u_{n}^{\prime}(r)\right| \leq \phi^{-1}\left(\frac{\lambda_{n} C}{c_{\lambda_{n}}^{\alpha} r^{N-1}} \int_{0}^{r} \frac{\tau^{N-1}}{\psi^{\alpha}} d \tau\right) \leq \phi^{-1}\left(\frac{\lambda_{n} C}{c_{\lambda_{n}}^{\alpha}(1-\alpha)}\right) \leq C_{1}
$$

for all $r \in(0,1)$ and $n$, where $C_{1}$ is a constant depending on $\lambda^{*}, C, \alpha, N, p$.

Hence $\left(u_{n}\right)$ is bounded in $C^{1}[0,1]$, and, by passing to a subsequence, we can assume that $u_{n} \rightarrow u_{\lambda^{*}}$ in $C[0,1]$. Letting $n \rightarrow \infty$ in

$$
u_{n}(r)=\int_{r}^{1} \phi^{-1}\left(\frac{\lambda_{n}}{s^{N-1}} \int_{0}^{s} \tau^{N-1} f\left(u_{n}\right) d \tau\right) d s
$$

we obtain

$$
u_{\lambda^{*}}(r)=\int_{r}^{1} \phi^{-1}\left(\frac{\lambda^{*}}{s^{N-1}} \int_{0}^{s} \tau^{N-1} f\left(u_{\lambda^{*}}\right) d \tau\right) d s
$$

i.e. $u_{\lambda^{*}}$ is a solution of $(1.2)$ with $\lambda=\lambda^{*}$. 
Lemma 5.4. Let $\lambda \in\left(0, \lambda^{*}\right)$ and let $u_{\lambda^{*}}$ be a solution of $(1.2)_{\lambda^{*}}$. Then there exists a positive number $\varepsilon$ such that $u_{\lambda^{*}}+\varepsilon$ is a supersolution of $(1.2)_{\lambda}$.

Proof. Let $p(x)=x^{\beta} f(x)$ and $\varepsilon_{0}=1-\lambda / \lambda^{*}$. Then there exists a positive number $\kappa_{0}$ such that $p \geq \kappa_{0}$ in $(0, \infty)$. Since $p$ is uniformly continuous on $\left(0,\left\|u_{\lambda^{*}}\right\|_{\infty}+1\right]$, there exists a number $\varepsilon \in(0,1)$ such that for all $x \in\left(0,\left\|u_{\lambda^{*}}\right\|_{\infty}\right]$,

$$
|p(x)-p(x+\varepsilon)|<\varepsilon_{0} \kappa_{0},
$$

hence

which implies

$$
\left|\frac{p(x)}{p(x+\varepsilon)}-1\right|<\varepsilon_{0}
$$

$$
\frac{f(x)}{f(x+\varepsilon)}=\frac{p(x)}{p(x+\varepsilon)}\left(1+\frac{\varepsilon}{x}\right)^{\beta}>1-\varepsilon_{0}=\frac{\lambda}{\lambda^{*}} .
$$

Consequently,

$$
-\left(r^{N-1} \phi\left(u_{\lambda^{*}}^{\prime}\right)\right)^{\prime}=\lambda^{*} r^{N-1} f\left(u_{\lambda^{*}}\right)>\lambda r^{N-1} f\left(u_{\lambda^{*}}+\varepsilon\right) \quad \text { in }(0,1),
$$

i.e., $u_{\lambda^{*}}+\varepsilon$ is a supersolution of $(1.2)_{\lambda}$.

Next, for each $v \in C[0,1]$, let $u=T_{\lambda} v$ be the solution of

$$
\left\{\begin{array}{l}
-\left(r^{N-1} \phi\left(u^{\prime}\right)\right)^{\prime}=\lambda r^{N-1}\left(g\left(\min \left(\max \left(v, \psi_{\lambda}\right), u_{\lambda^{*}}+\varepsilon\right)\right)+h\left(\min \left(v, u_{\lambda^{*}}+\varepsilon\right)\right),\right. \\
u^{\prime}(0)=0, \quad u(1)=0,
\end{array}\right.
$$

where $\varepsilon$ is defined in Lemma 5.4. Then $T_{\lambda}: C[0,1] \rightarrow C[0,1]$ is a compact operator and since

$$
\psi_{\lambda} \leq \min \left(\max \left(v, \psi_{\lambda}\right), u_{\lambda^{*}}+\varepsilon\right) \leq u_{\lambda^{*}}+\varepsilon,
$$

it follows from (A.3) that $T_{\lambda}$ is bounded.

Lemma 5.5. Every fixed point $u$ of $T_{\lambda}$ is a solution of (1.2) and satisfies

$$
\psi_{\lambda} \leq u \leq u_{\lambda^{*}}+\varepsilon \quad \text { in }[0,1] .
$$

Proof. Let $u$ be a fixed point of $T_{\lambda}$. Since $u_{\lambda^{*}} \geq \psi_{\lambda^{*}}>\psi_{\lambda}$, we have

$$
\left.g\left(\min \left(\max \left(u, \psi_{\lambda}\right), u_{\lambda^{*}}+\varepsilon\right)\right)\right)+h\left(\min \left(u, u_{\lambda^{*}}+\varepsilon\right)\right)=g\left(\max \left(u, \psi_{\lambda}\right)\right)+h(u) \geq \kappa
$$

if $u \leq u_{\lambda^{*}}+\varepsilon$, and

$$
\left.g\left(\min \left(\max \left(u, \psi_{\lambda}\right), u_{\lambda^{*}}+\varepsilon\right)\right)\right)+h\left(\min \left(u, u_{\lambda^{*}}+\varepsilon\right)\right)=f\left(u_{\lambda^{*}}+\varepsilon\right) \geq \kappa
$$

if $u \geq u_{\lambda^{*}}+\varepsilon$. This implies $u \geq \psi_{\lambda}$ in $(0,1)$, by Lemma 5.2(i). Suppose there exists $r_{0} \in(0,1)$ such that $u\left(r_{0}\right)>u_{\lambda^{*}}\left(r_{0}\right)+\varepsilon$. Then there exist numbers $r_{1}, r_{2}$ 
with $0 \leq r_{1}<r<r_{2}<1$ such that $u\left(r_{2}\right)=u_{\lambda^{*}}\left(r_{2}\right)+\varepsilon, u^{\prime}\left(r_{1}\right)=u_{\lambda^{*}}^{\prime}\left(r_{1}\right)$ or $u\left(r_{1}\right)=u_{\lambda^{*}}\left(r_{1}\right)+\varepsilon$, and $u>u_{\lambda^{*}}+\varepsilon$ on $\left(r_{1}, r_{2}\right)$.

Hence, by Lemma 5.4,

$$
-\left(r^{N-1} \phi\left(u^{\prime}\right)\right)^{\prime}=\lambda r^{N-1} f\left(u_{\lambda^{*}}+\varepsilon\right)<\lambda^{*} r^{N-1} f\left(u_{\lambda^{*}}\right)=-\left(r^{N-1} \phi\left(u_{\lambda^{*}}^{\prime}\right)\right)^{\prime}
$$

in $\left(r_{1}, r_{2}\right)$. Consequently,

$$
\begin{aligned}
0 & <\int_{r_{1}}^{r_{2}}\left(r^{N-1}\left(\phi\left(u^{\prime}\right)-\phi\left(u_{\lambda^{*}}^{\prime}\right)\right)\right)^{\prime}\left(u-\left(u_{\lambda^{*}}+\varepsilon\right)\right) d r \\
& =-\int_{r_{1}}^{r_{2}} r^{N-1}\left(\phi\left(u^{\prime}\right)-\phi\left(u_{\lambda^{*}}^{\prime}\right)\right)\left(u^{\prime}-u_{\lambda^{*}}^{\prime}\right) d r \leq 0,
\end{aligned}
$$

a contradiction. Thus $u \leq u_{\lambda^{*}}+\varepsilon$ in $(0,1)$, which completes the proof.

Proof of Theorem 2.2. Let $\lambda \in\left(0, \lambda^{*}\right)$. Since $\nu \phi_{1}$ is a subsolution of $(1.2)_{\lambda}$ if $\nu>0$ is sufficiently small and $u_{\lambda^{*}}$ is a supersolution of $(1.2)_{\lambda}$, it follows that (1.2) has a solution $u_{\lambda}$ such that $\nu \phi_{1} \leq u_{\lambda} \leq u_{\lambda^{*}}$. We shall show that $(1.2)_{\lambda}$ has a second solution. Define

$$
D=\left\{u \in C[0,1]:-\varepsilon<u<u_{\lambda^{*}}+\varepsilon \text { in }[0,1]\right\} .
$$

Then $D$ is an open set and $u_{\lambda} \in D$. By Lemma 5.5, all fixed points of $T_{\lambda}$ are in $\bar{D}$. Since $T_{\lambda}$ is bounded,

$$
\operatorname{deg}\left(I-T_{\lambda}, B\left(u_{\lambda}, R\right), 0\right)=1 \quad \text { for } R \gg 1 .
$$

If there exists $u \in \partial D$ such that $u=T_{\lambda} u$ then $u$ is a second solution of $(1.2)_{\lambda}$. Suppose that $u \neq T_{\lambda} u$ for all $u \in \partial D$. Then $\operatorname{deg}\left(I-T_{\lambda}, D, 0\right)$ is defined and since $T_{\lambda}$ has no fixed point in $B\left(u_{\lambda}, R\right) \backslash D$, it follows that

$$
\operatorname{deg}\left(I-T_{\lambda}, D, 0\right)=\operatorname{deg}\left(I-T_{\lambda}, B\left(u_{\lambda}, R\right), 0\right)=1 .
$$

Since $\tilde{A}_{\lambda}=T_{\lambda}$ on $D$, we have

$$
\operatorname{deg}\left(I-\tilde{A}_{\lambda}, D, 0\right)=1,
$$

and since by Lemma 5.2(i),

$$
\operatorname{deg}\left(I-\tilde{A}_{\lambda}, B\left(0, R_{0}\right), 0\right)=0
$$

for some $R_{0} \gg 1$, we arrive at

$$
\operatorname{deg}\left(I-\tilde{A}_{\lambda}, B\left(0, R_{0}\right) \backslash D, 0\right)=-1 .
$$

Thus there exists a fixed point $u$ of $\tilde{A}_{\lambda}$ in $B\left(0, R_{0}\right) \backslash D$, which is a second positive solution of (1.2). This completes the proof of Theorem 2.2. 


\section{Acknowledgements}

The author thanks the referee for carefully reading the manuscript and providing helpful suggestions.

\section{References}

[ANZ] W. Allegretto, P. Nistri, and P. Zecca, Positive solutions of elliptic nonpositone problems, Differential Integral Equations 5 (1992), 95-101. Zbl 0758.35032 MR 1141729

[Am1] H. Amann, On the number of solutions of asymptotically superlinear two point boundary value problems, Arch. Ration. Mech. Anal. 55 (1974), 207-213. Zbl 0294.34008 MR 0348178

[Am2] Fixed point equations and nonlinear eigenvalue problems in ordered Banach spaces, SIAM Rev. 18 (1976), 620-709. Zbl 0345.47044 MR 0415432

[AAB] A. Ambrosetti, D. Arcoya, and B. Buffoni, Positive solutions for some semipositone problems via bifurcation theory, Differential Integral Equations 7 (1994), 655-663. Zbl 0808.35030 MR 1270096

[AAP] A. Ambrosetti, J. Azoreno, and I. Peral, Multiplicity results for some nonlinear elliptic equations, J. Funct. Anal. 137 (1996), 219-242. Zbl 0852.35045 MR 1383017

[AA] C. Atkinson and K. E. Ali, Some boundary value problems for the Bingham model, J. Non-Newton. Fluid Mech. 41 (1992), 339-363. Zbl 0747.76012

[CP] M. M. Coclite ans G. Palmieri, On a singular nonlinear Dirichlet problem, Comm. Partial Differential Equations 14 (1989), 1315-1327. Zbl 0692.35047 MR 1022988

[CRT] M. G. Crandall, P. H. Rabinowitz, and L. Tartar, On a Dirichlet problem with a singularity, Comm. Partial Differential Equations 12 (1977), 193-222. Zbl 0362.35031 MR 0427826

[DMS] H. Dang, R. Manásevich, and K. Schmitt, Positive radial solutions of some nonlinear partial differential equations, Math. Nachr. 186 (1997), 101-113. Zbl :0881.34032 MR 1461215

[DSS] H. Dang, K. Schmitt, and R. Shivaji, On the number of solutions of boundary value problems involving the $p$-Laplacian, Electronic J. Differential Equations 1996, no. 1, 9 pp.

[DLN] D. G. DeFigueiredo, P.-L. Lions, and R. Nussbaum, A priori estimates and existence of positive solutions for semilinear elliptic equations, J. Math. Pures Appl. 61 (1982), 41-63. Zbl 0452.35030 MR 0664341

[Di] J. I. Díaz, Nonlinear partial differential equations and free boundaries, Pitman, London, 1985. Zbl 0595.35100 MR 0853732

[DHM] J. I. Díaz, J. Hernández, and F. J. Mancebo, Branches of positive and free boundary solutions for some singular quasilinear elliptic problems, J. Math. Anal. Appl. 352 (2009), 449-474. Zbl 1173.35055 MR 2499916

[DMO] J. I. Díaz, J. M. Morel, and 1. Oswald, An elliptic equation with singular nonlinearity, Comm. Partial Differential Equations 12 (1987), 1333-1344. Zbl 0634.35031 MR 0912208

[FM] W. Fulks and J. S. Maybee, A singular nonlinear equation, Osaka J. Math. 12 (1960), 1-19. Zbl 0097.30202 MR 0123095

[GMS] M. García-Huidobro, R. Manásevich, and K. Schmitt, Positive radial solutions of quasilinear elliptic partial differential equations on a ball, Nonlinear Anal. 35 (1999), 175-190. Zbl 0924.35047 MR 1643232 
[GS] B. Gidas and J. Spruck, A priori bounds for positive solutions of nonlinear elliptic equations, Comm. Partial Differential Equations 6 (1981), 883-901. Z Zbl 0462.35041 MR 0619749

[HS] D. D. Hai and K. Schmitt, On radial solutions of quasilinear boundary value problems, in Topics in nonlinear analysis, Progr. Nonlinear Differential Equations Appl. 35, Birkhäuser, Basel, 1999, 349-361. Zbl 0918.34027 MR 1725577

[HSS] D. D. Hai, K. Schmitt, and R. Shivaji, Positive solutions of quasilinear boundary value problems, J. Math. Anal. Appl. 217 (1998), 672-686. Zbl 0893.34017 MR 1492111

[HW] D. D. Hai and J. L. Williams, Positive radial solutions for a class of quasilinear boundary value problems in a ball, Nonlinear Anal. 75 (2012), 1744-1750. Zbl 1242.34040 MR 2870868

[HKS] J. Hernández, J. Karátson, and P. L. Simon, Multiplicity for semilinear elliptic equations involving singular nonlinearity, Nonlinear Anal. 65 (2006), 265-283. Zbl 05045904 MR 2228428

[HM] J. Hernández and F. J. Mancebo, Singular elliptic and parabolic equations, in Handbook of differential equations: Stationary partial differential equations, 2006, 317-400. Zbl 05679999

[LM] A. C. Lazer and P. J. McKenna, On a singular nonlinear elliptic boundary-value problem, Proc. Amer. Math. Soc. 111 (1991), 721-730. Zbl 0727.35057 MR 1037213

[LS1] V. Le and K. Schmitt, Global bifurcation in variational inequalities: Applications to obstacle and unitateral problems, Springer, New York, 1997. Zbl 0876.49008 MR 1438548

[LS2] _ On boundary value problems for degenerate quasilinear elliptic equations and inequalities, J. Differential Equations 144 (1998), 170-218. Zbl 0912.35069 MR 1615056

[Li] P.-L. Lions, On the existence of positive solutions of semilinear elliptic equations, SIAM Rev. 24 (1982), 441-467. Zbl 0511.35033 MR 0678562

[SY] J. Shi and M. Yao, On a singular nonlinear semilinear elliptic problem, Proc. Roy. Soc. Edinburgh Sect. A 128 (1998), 1389-1401. Zbl 0919.35044 MR 1663988

[SW] J. Smoller and A. Wasserman, Existence of positive solutions for semilinear elliptic equations in general domains, Arch. Ration. Mech. Anal. 98 (1987), 229-249. Zbl 0664.35029 MR 0867725

[St] C. A. Stuart, Existence and approximation of solutions of nonlinear elliptic equations, Math. Z. 147 (1976), 53-63. Zbl 0324.35037 MR 0404854

[TH] F. de Thelin and A. El Hachimi, Infinitely many radially symmetric solutions for a quasilinear elliptic problem in a ball, J. Differential Equations 128 (1996), 78-102. Zbl 0852.34021 MR 1392397

[Zh] Z. Zhang, On a Dirichlet problem with a singular nonlinearity, J. Math. Anal. Appl. 194 (1995), 103-113. Zbl 0834.35054 MR 1353070 